\title{
Making Sense of Mathematical Reasoning and Proof ${ }^{1}$
}

\author{
David Tall \\ University of Warwick \\ $<$ david.tall@warwick.ac.uk>
}

This paper presents a global theoretical framework that complements cognitive and affective aspects of the increasing sophistication of mathematical thinking and proof, taking into account the nature of mathematics itself and the way in which learners mature by building on their previous experiences. It is based on our shared human facilities of perception, action and reason that mature in very different ways, to explain and predict how we may develop mathematical thinking in general and mathematical reasoning and proof in particular.

\section{The sensori-motor language of mathematics}

The cognitive development of mathematical thinking and proof is based on fundamental human aspects that we all share: human perception, action and the use of language and symbolism that enables us to develop increasingly sophisticated thinkable concepts within increasingly sophisticated knowledge structures. It is based on what I term the sensori-motor language of mathematics, (Tall, forthcoming).

Mathematical thinking develops in the child as perceptions are recognised and described using language and as actions become coherent operations to achieve a specific mathematical purpose. According to Bruner (1966), these may be communicated first through enactive gestures, then iconic images, then the use of symbolism, including not only written and spoken language but also the operational symbolism of arithmetic and the axiomatic formal symbolism of logical deduction.

The theoretical framework proposed here follows a similar path enriched by the experience over time, building from conceptual embodiment that combines the enactive and iconic modes of human perception and action, developing into the mental world of perceptual and mental thought experiment. Embodied operations, such as counting, adding, sharing, are symbolised as manipulable concepts in arithmetic and algebra in a second mental world of operational symbolism. As the child matures, there is a further shift into a focus on the properties of mental objects as in Euclidean geometry, or the properties of arithmetic operations that are recast as 'rules' that underlie the generalized operations and expressions in algebra. Each of these leads to different forms of mathematical proof: Euclidean proof in geometry and symbolic proof, based on the 'rules of arithmetic' in arithmetic and algebra.

\footnotetext{
${ }^{1}$ This article is a product of personal experience, working with colleagues such as Shlomo Vinner who gave me the insight into the notion of concept image, Eddie Gray, whose experience with young children led me to grasp the essential ways in which children develop ideas of arithmetic and to build a theoretical framework for the different ways in which mathematical concepts are conceived, Michael Thomas who helped me understand more about how older children learn algebra, the advanced mathematical thinking group of PME who broadened my ideas about the different ways that undergraduates come to understand more formal mathematics, many colleagues and doctoral students who I celebrate in Tall (2008) and, more recently, the working group of ICMI 19 who focused on the cognitive development of mathematical proof (Tall, Yevdokimov et al., 2012).
} 
Embodiment and symbolism develop alongside each other and interact with each other. The early stages of practical mathematics begin with experience of shape and space, and of operations in arithmetic, in which properties of specific examples are seen to be generic, such as realising that $2+3=3+2$ holds not just for the numbers 2 and 3, but for any pair of whole numbers. This develops into the theoretical mathematics of definition and deduction in Euclidean and symbolic forms of proof.

Properties in both embodiment and symbolism develop into the formal mathematics of set-theoretic definition and proof in the axiomatic formal world of pure mathematics. While theoretical mathematics is based on embodied and symbolic experiences, formal mathematics guarantees that all the properties proved from given set-theoretic axioms and definitions will also hold in any new context that satisfies the given axioms and definitions.

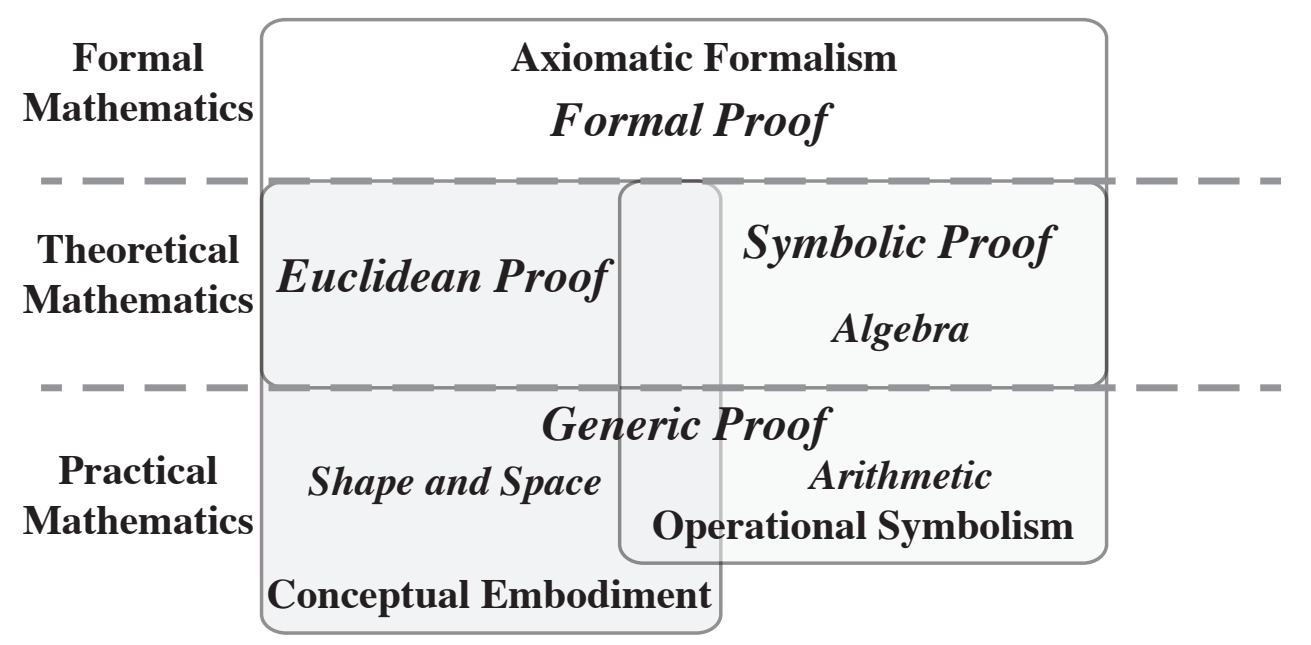

Figure 1: Outline of long-term development of proof

Embodiment and symbolism continue to play their part in axiomatic formalism, not only in imagining new possibilities that may be defined and proved formally, but also in an amazing turnaround in which certain theorems (called structure theorems) prove that axiomatic systems have embodied and symbolic structures established by formal proof. This reveals mathematical thinking at the highest level, and mathematical proof in particular, as an intimate blend of embodiment, symbolism and formalism where individual mathematicians develop a preference for different aspects.

\section{The evolution of theories of mathematical thinking and proof}

Pierre van Hiele (1986) focused on structure and insight, seeing a succession of levels that may be described as recognition and description of figures, leading to definition and deduction of properties through Euclidean proof.

Ed Dubinsky and others (Asiala et al, 1996) took an apparently different path, following Piaget's idea of reflective abstraction to focus on operations that are seen first as actions, routinized as processes, then encapsulated as mental objects within knowledge schemas. 
Anna Sfard (1991) proposed a framework that alternated between operational and structural ways of thinking in which operations are condensed as processes, and then reified as mental objects that now have a certain structure. She suggested at the time that an operational approach inevitably precedes structural mathematics. However, the examples given mainly involved operational symbolism becoming reified as mental objects, and there is a need to take account both of the van Hiele development of the properties of objects and the process-object encapsulation of operational symbolism.

This led to a three part analysis in Tall, Thomas et al. (2000) through parallel developments of conceptual embodiment (broadly following van Hiele) and operational symbolism (using process-object theories) in school, leading much later to the axiomatic formal framework of set-theoretic definition and proof in university pure mathematics (Tall, 2004a, 2004b).

Following the recent death of van Hiele in 2011 at the grand old age of one hundred, I revisited his ideas of structure and insight, which he asserted applied to geometry, but not to the symbolism of arithmetic and algebra (van Hiele, 2002). I realised that the term operation should not be restricted to the symbolic operations in arithmetic and algebra. Operations occur in the constructions of Euclidean geometry. For instance, we may operate on an isosceles triangle by joining the vertex to the midpoint of the base to cut the triangle into two parts that are congruent (with three corresponding sides). This proves that the base angles must be equal, and various other properties follow, such as the property that the line from the vertex to the midpoint of the base is at right angles to the base.

The operations of construction in geometry and the various operations in arithmetic and algebra have a common definition: they consist of 'a coherent sequence of actions and decisions performed to achieve a specific purpose.' A geometric operation is a construction that focuses on the object (the figure) and results in enabling us to see relationships concerning the properties of the object. A symbolic operation performs a calculation or manipulation, focusing more on the properties of the operations themselves as the operations lead to a symbolic output.

Furthermore the compression of operation into mental object in symbolism begins for the child as embodied operations on objects such as counting, adding, sharing, and is compressed into symbolic operations on whole numbers, fractions, signed numbers and so on. This reveals two distinct forms of compression from operation to mental object that I termed embodied compression and symbolic compression (Tall (forthcoming), chapter 7).

Embodied compression focuses on the effect of the operations on the objects, such as counting a collection to find the number of objects, such as 'six'. Focusing on the way that the objects are placed leads to a realisation of the fundamental properties of whole number arithmetic. For instance, the set of six objects may be subdivided, say, into subsets of 'four' and 'two' and, by rearranging the sets, it may be seen that 'two' and 'four' is also 'six'. Reorganizing the subsets as two rows of 'three' allows them to be seen as three columns of 'two' so that 'two threes' is the same as 'three twos'. 
Embodied compression enables us to see at a glance the flexible properties of arithmetic. 'Proof' at this early stage is a form of reasoning based on our interpretation of the coherence of our own perceptions and actions. This form of proof, in which a specific example is seen to be typical of a whole category of examples, is termed generic proof (Harel \& Tall, 1991).

Symbolic compression involves performing a counting operation to obtain a number concept, for instance, the operation of 'count-on' calculates 'two and eight' as counting on eight to get 'three, four, five, six, seven, eight, nine, ten' while 'eight and two' is the short count 'nine, ten'. Here the two operations are very different, one is a long count, and the other is short. The general properties of the symbolic compression are therefore not as self-evident as they are with embodied compression.

A gifted child may grasp the flexible properties of arithmetic as part of a coherent knowledge structure that may be used as an organising principle to simplify operations. A child who focuses on procedural operations of counting taking place in time will find arithmetic operations to be far more difficult to cope with. Eddie Gray and I called this bifurcation 'the proceptual divide' between those fixed in increasingly complicated counting procedures and those who develop flexible ways to derive new facts from known facts (Gray \& Tall, 1994).

This bifurcation between those who find mathematics 'easy' and those who find it impossibly difficult begins at a very early age. It should be taken into account in seeking to explain and predict how each individual attempts to make sense of mathematics by building on personal ways of knowing, operating and proving.

\section{Long-term pleasure and pain}

Emotions play a vital role in mathematical thinking and have a profound effect on how individuals make sense of mathematical proof. As my supervisor, Richard Skemp used to say: 'pleasure is a signpost, not a destination.' His goal-oriented theory of learning (Skemp 1979) saw children starting out with the goal of seeking to make sense of the world. Successfully linking together ideas in coherent ways gives pleasure, success breeds more success, so that a child with a history of success builds up a positive feed-back loop where an encounter with a problematic situation is often met with the determination to conquer the difficulty. However, lack of success leads to an anti-goal, to avoid the feeling of stress. Further encounters with stress may lead to a negative feed-back loop in which the desire to avoid failure leads to less engagement with the mathematics and less technical proficiency that causes even more difficulty and greater mathematical anxiety (Baroody \& Costlick, 1998).

An analysis of the development of mathematical thinking reveals the surprising conclusion that mathematics is not a system that builds logically on previous experience at each stage, even though every mathematics curriculum in the world is intent on presenting topics in a coherent sequence, carefully preparing the necessary pre-requisites at each stage for the more sophisticated stages that follow. On the contrary, an experience that has been 'met before' may be supportive in some new situations yet problematic in others. 
The concept of 'met-before' was introduced by Lima \& Tall, (2008) and McGowen \& Tall (2010) to describe 'a structure we have in our brains now as a result of experiences we have met before.' Some ideas that work in one situation such as 'addition makes bigger' or 'take away makes smaller' in whole number arithmetic are supportive in the context of fractions yet problematic in the context of signed numbers. This recalls the concept of 'epistemological obstacle' developed by Bachelard (1938) and Brousseau (1983) and the need for accommodation by Piaget (see, for example, Baron et al, 1995) or reconstruction by Skemp (1971).

However, the notion of met-before refers to the effect of previous experience on new learning. A particular met-before is not in itself supportive or problematic, it becomes supportive or problematic in a new situation when the learner attempts to make sense of the new ideas. For instance, 'take away leaves less' is supportive in some contexts (e.g. everyday situations where something is removed, in the postulates of Euclidean geometry, or taking one whole number from another) but it is problematic in others (such as taking away a negative number or in the theory of infinite cardinals).

A problematic met-before arises not only in the individual learner, it is a widespread feature of the nature of mathematics itself. In shifting to a new context, say from whole numbers to fractions, or from positive numbers to signed numbers, or from arithmetic to algebra, generalization is encouraged by supportive met-befores (ideas that worked in a previous context and continue to work in the new one) and impeded by problematic met-befores (that made sense before but do not work in the new context).

These subtle changes of meaning arise as mathematical ideas are generalized. Properties such as commutativity, associativity, distributivity are supportive as number systems are broadened through whole numbers, integers, real numbers, complex numbers, but other aspects such as 'take away gives less' or 'the square of a non-zero number is positive' become problematic and impede progress.

\section{Crystalline concepts}

Given this increasing difficulty of problematic aspects that occur in generalization, I sought a unifying principle that is supportive in mathematical thinking and binds mathematical ideas together in any given context. In Tall (2011), I formulated a working definition of a crystalline concept as 'a mathematical concept that has an internal structure of relationships that cause it to have specific properties in the given mathematical context.' Such concepts include:

- platonic objects in geometry, such as points, lines, triangles, circles, congruent triangles, parallel lines that have properties arising through Euclidean proof;

- operational symbols as flexible procepts in arithmetic, algebra and symbolic calculus that have necessary properties through calculation and manipulation;

- set-theoretically defined concepts in axiomatic formal mathematics whose properties are deduced by formal proof. 
Not only do crystalline concepts occur at the highest levels of mathematical thinking, they emerge in the thinking of a young child who sees the flexible proceptual structure of arithmetic through embodied compression rather than the procedural step-by-step counting procedures of arithmetic that operate in time.

They enable flexible thinkers to see mathematical ideas in astonishingly simple ways. It is not that the fractions $4 / 8,7 / 14,101 / 202$ are all equivalent to each other and to the simplest possible canonical form $1 / 2$, it is that they are all manifestations of a single crystalline concept: the rational number as a unique point on the number line.

It is not that the expressions $2(x+7)$ and $2 x+14$ are equivalent but different, where the first can be turned into the second by 'multiplying out the brackets' and the second can be turned into the first by 'factorization', it is that both expressions are different ways of writing the same crystalline concept as an algebraic expression. Indeed, the functions $f(x)=2(x+7)$ and $g(x)=2 x+14$ are not simply equivalent, they are precisely the same function. Students who think flexibly in terms of crystalline concepts have much more powerful means of relating mathematical ideas than those who see equivalent ideas that are changed from one form to another by carrying out procedures.

Likewise, in axiomatic formal mathematics, an axiomatic system such as 'a group' is a crystalline concept with rich interconnections between its properties. We may not know what specific group we are dealing with, but we do know that it has an identity that we may denote by $e$, and that if $x$ is any element, we can define the power $x^{n}$ for any positive or negative integer and prove that $x^{m+n}=x^{m} x^{n}$ for any integers $m, n$.

A crystalline concept may be defined formally and then its properties may be deduced as theorems to build up a knowledge structure where relationships are tightly interconnected by formal proof. For example, we can prove that if we begin with the axiomatic definition of an ordered field $F$, then in this context we may formulate any of the equivalent definitions for completeness, to prove that a complete ordered field is not only unique up to isomorphism, it is also unique as a crystalline concept.

At the highest level of pure mathematical research, it is the compression of structural properties of defined formal concepts into crystalline concepts that gives gifted mathematicians a simplicity of thought that is beyond the mere proving of theorems of equivalence. An ordered field not only contains a subfield isomorphic to the rational numbers, it can be conceived as a crystalline concept that contains the crystalline concept of the rational numbers.

I recall the ideas that I encountered as a graduate student when theoreticians spoke of the identification of one structure with another structure as 'an abuse of notation'. On the contrary, it is the very vision that gives the biological brain of the mathematician a level of flexibility to conceive mathematical ideas mentally in more simple and insightful ways. 


\section{The transition from proof in embodiment and symbolism to formal proof}

The overall framework for cognitive development from the newborn child to the frontiers of mathematical research was further developed in the ICMI Project on Proof and Proving (Tall, Yevdokimov et al, 2012), and has been extended in How Humans Learn to Think Mathematically (Tall, forthcoming).

The van Hiele levels (1986) have been variously reconsidered by a range of authors, may now be seen in as four successive levels which I term

- Recognition of basic concepts such as points, lines, and various shapes;

- Description of observed properties;

- Definition of concepts to test new examples to see if they satisfy the definition and to use the definitions to formulate geometric constructions;

- Deduction in the form of Euclidean proof in plane geometry.

Each of these is a form of structural abstraction in which the structure of the objects under consideration and their relationships shift to successive new levels of sophistication. This begins first with observations of geometric objects whose structures are recognised and described. At this point the foundations of Euclidean proof are laid down by formulating definitions for figures that not only allow them to be categorised and constructed but also to use ideas such as congruent triangles and parallel lines to construct Euclidean proof.

Van Hiele also described a fifth level of rigour that may be seen as shifting in two directions, the first is to different embodied contexts such as projective geometry or spherical geometry, the second is in terms of the more sophisticated world of axiomatic formalism as prescribed by Hilbert.

Van Hiele (2002) saw these levels apply to geometry and not to the symbolic development from arithmetic to algebra. The calculation with numbers and manipulation of algebraic symbols involve quite different mental activities from those in Euclidean proof. However, once operations are encapsulated as number concepts and generalized as algebraic expressions, these too have properties that can be recognised and described, then defined as 'rules of arithmetic' to be used in algebraic proofs to deduce theorems. Thus the sequence of structural abstraction also occurs in the higher levels of operational symbolism to provide definitions of whole numbers, such as even, odd, prime and to deduce theorems such as the uniqueness of factorization into primes.

Exactly the same structural abstraction arises in the axiomatic formal world of settheoretic definition and formal proof. This builds on our experience of conceptual embodiment and operational symbolism, beginning with the recognition and description of mathematical situations and then the definition of axiomatic systems and of defined concepts within those systems, and deduction of properties of systems and defined concepts using formal proof.

Experienced mathematicians have flexible knowledge structures that they wish to pass on to their students. However, by the time students pass through school to enter 
university, they will have already developed in very different ways based on how they have managed to make sense of previous experiences.

Krutetskii (1976) produced significant evidence that the most gifted children are more likely to develop a strong verbal-logical basis to mathematical thinking than a visual-pictorial foundation. Out of over a thousand students, the most gifted nine were classified with five analytic (verbal logical), one geometric (visual-pictorial), two combining both (one more visual, the other more verbal) and one who was not classified. Presmeg (1986) found that the most outstanding senior school mathematics students in her study (7 pupils out of 277) were almost always nonvisualizers. Of 27 'very good' students (10\% of the sample), eighteen were nonvisualizers and five were visualizers.

This suggests that a small number of those students who enter university are powerful verbal-analytic thinkers who may benefit from making sense of settheoretic definitions, an even smaller number base their thinking on visual-pictorial representations, and many others with a blend of visual embodied thinking and operational symbolism.

Some students seek a natural approach based on a blend of previous experiences of embodiment and symbolism from school mathematics. Some with a more verballogical basis may seek to use a formal approach based on set-theoretic definitions and the deduction of properties using formal proof. Others seek to learn proofs procedurally to reproduce in examinations. All of these approaches may involve supportive and problematic aspects, which have been detailed in the literature (e.g. Pinto \& Tall, 1999, Weber, 2004).

As students become more experienced and shift to graduate studies, Weber (2001) produced evidence that research graduates are more likely to respond flexibly problems by making links between concepts in a sophisticated knowledge structure while undergraduates in their early studies, have yet to develop such flexibility.

This is consistent with the lack of aesthetic appreciation of mathematical ideas noted by Dreyfus and Eisenberg (1986) and also with the relationship noted by Koichu, Berman \& Katz (2007) between "aesthetical blindness" of students and factors such as self-esteem that affect their aesthetic judgement.

The theoretical framework presented here traces the development of cognitive and emotional aspects throughout the lifetime of the individual. A few students, characterized as being 'gifted' develop verbal-analytic skills that enable them to build formally from set-theoretic definitions to construct highly connected crystalline concepts that may have embodiments and operations linked to underlying formally proved structure theorems. But many others, who focus on 'maximising their mark on the exam' to 'get a good degree' to move on in their lives, have good reasons for doing so. The mathematics is problematic for them and it doesn't make sense.

\section{Structure Theorems}

Some theorems based on formal axioms and definitions prove formal structures that enable the ideas to be reconsidered in embodied and symbolic terms. For example, a 
finite dimensional vector space over a field $F$ is isomorphic to $F^{n}$, so that its elements may be represented symbolically as $n$-tuples and its linear maps as matrices, and in the case where $F$ is the field of real numbers and $n=2$ or 3, it may be embodied in two or three dimensional space. In the same way a finite group is isomorphic to a subgroup of a group of permutations, which allows it to be operated on symbolically and embodied as the transformations of a geometric object.

Structure theorems enrich formal mathematics with new forms of embodiment and symbolism, offering a wide range of embodiment, symbolism and formalism to enable mathematicians to explore new situations that follow a sequence of structural abstraction, beginning with the recognition and description of a problem situation, then the definition of conjectures and a quest for the deduction of new theorems.

\section{The overall development of proof}

Long-term the growth of mathematical thinking of proof begins with the perceptions and actions of young children, and develops through three successive levels:

- practical mathematics exploring shape and space and developing experience of the operations of arithmetic. This involves the recognition and description of properties, such as the observation that the sum of numbers is not affected by the order of operation.

- theoretical mathematics of definition and deduction, as exhibited by Euclidean proof in geometry, and of the definition of the 'rules of arithmetic' and properties such as even, odd, prime composite, and the deduction of theorems.

Theoretical mathematics is appropriate for most applications of mathematics, while those who go on to study pure mathematics change meaning once more to

- formal mathematics based on set-theoretic definition and deduction.

In mathematical research, mathematicians use a combination of embodiment, symbolism and formalism to imagine possible theorems and to formulate conjectures to seek proof and to shift to ever more sophisticated levels using structure theorems.

The framework offers mathematicians, mathematics educators, teachers and learners the opportunity to share an overall development of proof based on the fundamental sensori-motor bases of human thinking that becomes increasingly sophisticated through the use of language and symbolism. It offers an integration of the cognitive and affective development of mathematical knowledge and mathematical proof. This may be blended with aspects of interest in other communities of practice involved in mathematics, science, sociology, psychology, philosophy, history, cognitive science, constructivism and so on.

It begins with practical experiences in which specific examples may be seen as generic examples of proof. Then these experiences lead to theoretical proof based on definition and Euclidean proof in geometry and definitions based on the 'rules of 
arithmetic' in arithmetic and algebra. At a formal level, definitions are given as quantified set-theoretic definitions and formal proof that apply in any context where the axioms and definitions are satisfied.

The long-term development is affected by supportive and problematic met-befores that apply not only to developing students, but also to the historical evolution of mathematics and to the competing views of differing communities of practice. Experts with sophisticated knowledge structures are subject to personal conceptions of mathematics that they may share with other experts in their community but perhaps not with other communities. The framework offers an opportunity to evolve theoretical ideas into the future by blending differing viewpoints with the long-term development of mathematical thinking through perception, operation and reason.

\section{References}

Asiala, M., Brown, A., DeVries, D., Dubinsky, E., Mathews, D., Thomas, K. (1996). A framework for research and curriculum development in undergraduate mathematics education, Research in Collegiate Mathematics Education II, CBMS Issues in Mathematics Education. 6, 1996, 1-32.

Bachelard, G. (1938), (reprinted 1983), La formation de l'esprit scientifique, J. Vrin, Paris.

Baron, R., Earhard, B., \& Ozier, M. (1995). Psychology (Canadian Edition). Scarborough, ON: Allyn \& Bacon.

Baroody, A.J. \& Costlick, R.T. (1998). Fostering children's mathematical power. An investigative approach to K-8 mathematics instruction. New Jersey: Lawrence Erlbaum Associates, Inc.

Brousseau, G. (1983), Les obstacles épistémologiques et les problèmes en mathématiques, Recherches en didactique des Mathématiques, 4(2) 164-198.

Bruner, J. S. (1966). Towards a Theory of Instruction, New York: Norton.

Dreyfus, T. \& Eisenberg, T. (1986). On the aesthetics of mathematical thoughts. For the Learning of Mathematics, 6 (1), 2-10.

Gray, E. M. \& Tall, D. O. (1994). Duality, Ambiguity and Flexibility: A Proceptual View of Simple Arithmetic, Journal for Research in Mathematics Education, 26 2, 115-141.

Harel, G. \& Tall, D. O. (1991). The General, the Abstract, and the Generic in Advanced Mathematics, For the Learning of Mathematics, 11 1, 38-42.

Koichu, B. (2008). On considerations of parsimony in mathematical problem solving. In O. Figueras, J.L. Cortina, S. Alatorre, T. Rojano \& A. Sepulova (Eds.), Proceedings of the 32nd Conference of the International Group for the Psychology of Mathematics Education, Vol. 3, pp. 273-280, Morelia, Mexico.

Koichu, B., Berman, A. \& Katz, E. (2007). What is a beautiful problem? An undergraduate students' perspective. In Woo, J. H., Lew, H. C., Park, K. S. \& Seo, D. Y. (Eds.). Proceedings of the 31st Conference of the International Group for the Psychology of Mathematics Education, Vol. 3, pp. 113-120. Seoul: PME.

Krutetskii, V. A., (1976). The Psychology of Mathematical Abilities in Schoolchildren, University of Chicago Press, Chicago.

Lima, R. N. de, \& Tall, D. O. (2008). Procedural embodiment and magic in linear equations. Educational Studies in Mathematics, 67 (1), 3-18.

McGowen, M. C. \& Tall, D. O. (2010). Metaphor or Met-before? The effects of previous 
experience on the practice and theory of learning mathematics. Journal of Mathematical Behavior 29, 169-179.

Pinto, M. M. F. \& Tall, D. O. (1999), Student constructions of formal theory: giving and extracting meaning. In O. Zaslavsky (Ed.), Proceedings of the 23rd Conference of $P M E$, Haifa, Israel, 4, 65-73.

Presmeg, N. C. (1986). Visualisation and Mathematical Giftedness. Educational Studies in Mathematics, 17 (3), 297-311.

Sfard, A. (1991). On the Dual Nature of Mathematical Conceptions: Reflections on processes and objects as different sides of the same coin, Educational Studies in Mathematics, 22, 1-36.

Skemp, R. R. (1971). The Psychology of Learning Mathematics. Penguin: London.

Skemp, R. R. (1979). Intelligence, Learning, and Action. Wiley: London.

Tall, D. O, (2004a). The three worlds of mathematics. For the Learning of Mathematics, 23 (3). 29-33.

Tall, D. O. (2004b). Thinking through three worlds of mathematics, Proceedings of the 28th Conference of the International Group for the Psychology of Mathematics Education, Bergen, Norway, 4, 281-288.

Tall, D. O. (2008). A life-time's journey from definition and deduction to ambiguity and insight. Mediterranean Journal for Research in Mathematics Education, 7 (2), 183196.

Tall, D. O. (2011) Crystalline concepts in long-term mathematical invention and discovery. For the Learning of Mathematics, 31 (1) March 2011, 3-8.

Tall, D. O. (forthcoming). How Humans Learn to Think Mathematically. New York: Cambridge University Press. (See http://www.davidtall.com/threeworlds/ for the latest information.)

Tall, D. O., Lima, R. N., \& Healy, L. (under review). Evolving a three-world framework for solving algebraic equations in the light of what a student has met before. (Available from http://www.warwick.ac.uk/staff/David.Tall/downloads.html)

Tall, D. O., Thomas, M. O. J., Davis, G., Gray, E. M., Simpson, A. P. (2000). What is the object of the encapsulation of a process? Journal of Mathematical Behavior, 18 (2), $1-19$.

Tall, D. O, Yevdokimov, O., Koichu, B., Whiteley, W., Kondratieva, M., Cheng, Y. H. (2012). Cognitive Development of Proof. In ICMI 19: Proof and Proving in Mathematics Education. Springer.

Van Hiele, P. (1986). Structure and Insight. Orlando: Academic Press.

Van Hiele, P. (2002). Similarities and Differences between the Theory of Learning and Teaching of Skemp and the Van Hiele Levels of Thinking. In Tall, D. O. \& Thomas, M. O. J. (Eds), Intelligence, Learning and Understanding - A Tribute to Richard Skemp (pp. 27-47). Post Pressed, Flaxton, Australia.

Weber, K. (2001). Student difficulty in constructing proofs: the need for strategic knowledge. Educational Studies in Mathematics, 48 (1), 101-119.

Weber, K. (2004). Traditional instruction in advanced mathematics courses: a case study of one professor's lectures and proofs in an introductory real analysis course, Journal of Mathematical Behavior, 23, 115-133. 